\title{
An accurate treatment of diffuse reflection boundary conditions for a stochastic particle Fokker-Planck algorithm with large time steps
}

\author{
Thomas Önskog ${ }^{\mathrm{a}, *, 1}$, Jun Zhang ${ }^{\mathrm{b}, \mathrm{c}}$ \\ a Department of Mathematics, Stockholm University, SE-106 91 Stockholm, Sweden \\ b James Weir Fluids Laboratory, Department of Mechanical and Aerospace Engineering, University of Strathclyde, Glasgow G1 1XJ, \\ United Kingdom \\ c State Key Laboratory of High Temperature Gas Dynamics, Institute of Mechanics, Chinese Academy of Sciences, Beijing 100190, China
}

\section{H I G H L I G H T S}

- A stochastic particle algorithm for simulation of flows of wall-confined gases is presented.

- The algorithm is constructed for large time steps which reduces the computational cost.

- Numerical simulations verify that the proposed algorithm reproduces the correct boundary behaviour.

\section{A R T I C L E I N F O}

\section{Article history:}

Received 10 February 2015

Received in revised form 24 April 2015

Available online 21 August 2015

\section{Keywords:}

Rarified gas flows

Fokker-Planck equation

Langevin simulation

Boundary conditions

Stochastic differential equations

First hitting time

\begin{abstract}
A B S T R A C T
In this paper, we present a stochastic particle algorithm for the simulation of flows of wall-confined gases with diffuse reflection boundary conditions. Based on the theoretical observation that the change in location of the particles consists of a deterministic part and a Wiener process if the time scale is much larger than the relaxation time, a new estimate for the first hitting time at the boundary is obtained. This estimate facilitates the construction of an algorithm with large time steps for wall-confined flows. Numerical simulations verify that the proposed algorithm reproduces the correct boundary behaviour.
\end{abstract}

(C) 2015 Elsevier B.V. All rights reserved.

\section{Introduction}

At present, the most widely used particle method for simulating gas flows is the direct simulation Monte Carlo (DSMC) method [1] proposed by Bird in the 1960s. The fundamental idea behind the DSMC method is to track a large number of representative molecules, with their motions and inter-molecular collisions assumed uncoupled. Molecular motions are modelled deterministically according to the Newtonian equations of motion, while molecular collisions are modelled statistically by selecting collision pairs in cells. To correctly reproduce the transport properties of gases, the sizes of the cells within which molecular collision partners are selected must not exceed the mean free path of molecules, and the time steps

\footnotetext{
* Corresponding author.

E-mail addresses: onskog@kth.se (T. Önskog), zhangjun04@foxmail.com (J. Zhang).

1 Current address: Department of Mathematics, KTH Royal Institute of Technology, SE-100 44 Stockholm, Sweden.
} 
should be less than the mean collision time [2,3]. Therefore, the DSMC method becomes very expensive for simulation of small Knudsen number flows.

In contrast, the particle Fokker-Planck model uses a Langevin equation to describe a continuous stochastic process in velocity space. The velocities of each particle are separately updated according to the drag force and stochastic force, and no individual particle collisions need to be considered. This allows the sizes of cells and time steps to be chosen independently of the mean free path and mean collision time, respectively. Accordingly, for the simulation of small Knudsen number flows, the computational efficiency is much higher than for the DSMC method. A stochastic particle algorithm for solving the particle Fokker-Planck model was proposed by Jenny et al. [4]. Since then, great progress has been made and the applications have been extended to a variety of gas flows [5-7].

A general review of Langevin simulation of gas flows was presented in Ref. [8], where two critical issues of such simulations are discussed. The first issue is related to the transport properties of Langevin models. Using the Green-Kubo formula, Zhang et al. [9] obtained analytical expressions for the transport coefficients, including the diffusion, viscosity and thermal conductivity coefficients. It was shown that the simple Langevin model predicts a false Prandtl number for gas molecules. This problem could, however, be solved using the cubic nonlinear Langevin model proposed by Gorji et al. [7] and the Langevin acceleration model proposed by Heinz [10,11].

The second issue concerns boundary conditions. In the absence of boundary walls, the Langevin model proposed in Ref. [4] is statistically exact for constant macroscopic velocity and energy for any size of the time steps. If a boundary wall is present, some particles will, in each calculating time step, hit the wall during the stochastic diffusion process. To employ boundary conditions, it is very crucial to determine as exactly as possible when the particles hit the boundary. In Ref. [4], a simple linear interpolation method was used to obtain the hitting time. However, this approximation is only accurate in the limit of very small time steps. According to the analysis of the Langevin equation carried out in the article at hand, two distinct characteristics exist in the short time and long time limits, respectively. In the short time limit, the movement of the particles is free, and hence the mean displacement is linear in time. In this case, it is reasonable to use linear interpolation to determine the hitting time. In the long time limit, on the other hand, the movement of the particles is a diffusion process and the mean displacement is proportional to the square root of time. Therefore, linear interpolation is no longer applicable for large time steps. As shown in Section 4, the scheme of Ref. [4] with linear interpolation predicts a higher density close to the wall. This effect is due to linear interpolation overestimating the hitting time and, consequently, underestimating the remaining time after the boundary hit. This implies that the simulated particles do not have sufficient time to move away from the boundary and, thus, more particles are found close to the wall. In this article we address the problem of determining the hitting time accurately, as this is the main remaining obstacle for constructing an efficient Langevin model with large time steps for wall-confined flows.

The DSMC method is very efficient for flows with large Knudsen number $(\mathrm{Kn}>0.1)$, and the particle Fokker-Planck model proposed in Refs. [4,7] is efficient for flows with moderate Knudsen number $(0.01<\mathrm{Kn}<0.1)$. Our aim is to develop an efficient particle Fokker-Planck model for flows with small Knudsen number $(\mathrm{Kn}<0.01)$ using large time steps. In this article, we consider one-dimensional, wall-confined flows with zero macroscopic velocity and no external forces and we postpone the more general case of multi-dimensional flows with nonzero macroscopic velocity to a future article.

This article is arranged as follows. In Section 2, we present some basic mathematical results for the Langevin model and derive an estimate for the first hitting time of the boundary in the limit of large time steps. In Section 3, we present a new stochastic particle Fokker-Planck algorithm using the first hitting time estimate from Section 2. Simulation results for a particular wall-confined flow are demonstrated in Section 4 and some conclusions are presented in Section 5.

\section{Mathematical analysis of the stochastic model}

In this section we provide a mathematical basis for the algorithm proposed in this article. In the absence of a macroscopic velocity and external forces, the solution to the Fokker-Planck equation can be transformed into the equivalent Itō processes $X_{t}$ and $M_{t}$ satisfying

$$
\begin{aligned}
& \mathrm{d} X_{t}=M_{t} \mathrm{~d} t, \\
& \mathrm{~d} M_{t}=-\frac{1}{\tau} M_{t} \mathrm{~d} t+\sqrt{\frac{4 e_{s}}{3 \tau}} \mathrm{d} W_{t},
\end{aligned}
$$

for $t \geq 0$, with initial conditions $X_{0}=x$ and $M_{0}=m$, see for example Ref. [4]. Here $\tau$ is the relaxation time, that is the average time between two particle collisions, and $e_{s}$ is the average kinetic energy of particles. The process $X_{t}$ can be interpreted as the position and the process $M_{t}$ as the velocity of a particle moving along the flow. Using Itō calculus on $X_{t}$ and $M_{t}$, it is straightforward to verify that, given the location $X_{t_{n}}$ and velocity $M_{t_{n}}$ at some time $t_{n}$, the position and velocity evolves according to

$$
\begin{aligned}
& X_{t_{n}+t}=X_{t_{n}}+M_{t_{n}} \tau\left(1-\mathrm{e}^{-t / \tau}\right)+\sqrt{\frac{4 e_{s} \tau}{3}} \int_{0}^{t}\left(1-\mathrm{e}^{(s-t) / \tau}\right) \mathrm{d} W_{s}, \\
& M_{t_{n}+t}=M_{t_{n}} \mathrm{e}^{-t / \tau}+\sqrt{\frac{4 e_{s}}{3 \tau}} \int_{0}^{t} \mathrm{e}^{(s-t) / \tau} \mathrm{d} W_{s},
\end{aligned}
$$


for $t>0$, in the absence of a macroscopic velocity. The correlations between $X_{t}$ and $M_{t}$ conditioned on the history of the processes up to time $t_{n}$ are

$$
\begin{aligned}
& E\left[\left(X_{t_{n}+t}-X_{t_{n}}\right)^{2} \mid \mathcal{F}_{t_{n}}\right]=M_{t_{n}}^{2} \tau^{2}\left(1-\mathrm{e}^{-t / \tau}\right)^{2}+\frac{2 e_{s} \tau^{2}}{3}\left(\frac{2 t}{\tau}-\left(3-\mathrm{e}^{-t / \tau}\right)\left(1-\mathrm{e}^{-t / \tau}\right)\right), \\
& E\left[M_{t_{n}+t}^{2} \mid \mathcal{F}_{t_{n}}\right]=M_{t_{n}}^{2} \mathrm{e}^{-2 t / \tau}+\frac{2 e_{s}}{3}\left(1-\mathrm{e}^{-2 t / \tau}\right),
\end{aligned}
$$

and

$$
E\left[\left(X_{t_{n}+t}-X_{t_{n}}\right) M_{t_{n}+t} \mid \mathcal{F}_{t_{n}}\right]=M_{t_{n}}^{2} \tau \mathrm{e}^{-t / \tau}\left(1-\mathrm{e}^{-t / \tau}\right)+\frac{2 e_{s} \tau}{3}\left(1-\mathrm{e}^{-t / \tau}\right)^{2},
$$

as was previously stated in Ref. [4]. In this article, we consider wall-confined flows and we want the position process to satisfy $X_{t_{n}+t} \in\left[L_{1}, L_{2}\right]$, for $t>0$, with diffuse reflection at the boundaries. To accomplish this, we need to determine if and when $X_{t_{n}+t}$ hits the boundary, and we do this, by first investigating $X_{t_{n}+t}$ in the limits of very small and very large values of $t$, respectively.

For $t \ll \tau$, a Taylor expansion of (2.5) in the variable $t / \tau$ shows that the location $X_{t_{n}+t}$ is normally distributed with mean $X_{t_{n}}+M_{t_{n}} t$ and variance $4 e_{s} t^{3} / 9 \tau$. If $M_{t_{n}}^{2}$ is of the same order as $e_{s}$, which it should be in the mean as we have $E\left[M_{t_{n}}^{2}\right]=2 e_{s} / 3$, then the stochastic part is negligible compared to the deterministic part and $X_{t_{n}+t} \approx X_{t_{n}}+M_{t_{n}} t$. This corresponds to the decoupling of the velocity and position updates for small time steps in the numerical algorithm proposed in Ref. [6].

For $t \gg \tau$, the exponential terms in (2.5) are insignificant. Note for example that already for $t \approx 4 \tau$ the relative contribution of the exponential terms to the variance of the location is of the order $10^{-2}$. Hence, for large $t$, the location $X_{t_{n}+t}$ is normally distributed with mean $X_{t_{n}}+M_{t_{n}} \tau$ and variance $2 e_{s} \tau(2 t-3 \tau) / 3$. It is interesting to note here that the mean change in location $X_{t_{n}}+M_{t_{n}} \tau$ is independent of $t$ and occurs on a time scale of size $\tau$, whereas the stochastic change in location increases in time at the same rate as for a Wiener process. For large $t$, we could hence model $X_{t_{n}+t}$ as

$$
X_{t_{n}+t}=X_{t_{n}}+M_{t_{n}} \tau+\sqrt{\frac{4 e_{s} \tau}{3}} \widetilde{W}_{t-3 \tau / 2}
$$

for some standard Wiener process $\widetilde{W}$.

\subsection{First hitting times for Wiener processes}

As we are interested in the boundary behaviour of $X_{t_{n}+t}$ and we have seen above that for $t \gg \tau$, the process $X_{t_{n}+t}$ behaves like a Wiener process, we shall derive a few results regarding hitting times for Wiener processes. Let $W_{t}^{a}$ denote a Wiener process with variance $\sigma^{2}$ starting at location $a>0$ at time zero. The first hitting time $T^{a}$ of $W_{t}^{a}$ at zero is defined as $T^{a}=\inf \left\{t>0: W_{t}^{a}=0\right\}$ and has the well-known density

$$
P\left(T^{a} \in \mathrm{d} t\right)=\frac{a}{\sqrt{2 \pi \sigma^{2} t^{3}}} \exp \left(-\frac{a^{2}}{2 \sigma^{2} t}\right) \mathrm{d} t .
$$

The Wiener process has independent increments so the joint density of $T^{a}$ and $W_{T}^{a}$, for $T^{a} \leq T$, is simply given by

$$
P\left(T^{a} \in \mathrm{d} t, W_{T}^{a} \in \mathrm{d} b\right)=P\left(T^{a} \in \mathrm{d} t\right) P\left(W_{T-t}^{0} \in \mathrm{d} b\right) .
$$

Conditioning on the location of the Wiener process at time $T$, we obtain, using the well-known density of a Wiener process with drift $\sigma$,

$$
\begin{aligned}
P\left(T^{a} \in \mathrm{d} t \mid W_{T}^{a}=b\right) & =\frac{P\left(T^{a} \in \mathrm{d} t, W_{T}^{a} \in \mathrm{d} b\right)}{P\left(W_{T}^{a} \in \mathrm{d} b\right)}=\frac{P\left(T^{a} \in \mathrm{d} t\right) P\left(W_{T-t}^{0} \in \mathrm{d} b\right)}{P\left(W_{T}^{a} \in \mathrm{d} b\right)} \\
& =a \sqrt{\frac{T}{2 \pi \sigma^{2}(T-t) t^{3}}} \exp \left(\frac{(a-b)^{2}}{2 \sigma^{2} T}-\frac{a^{2}}{2 \sigma^{2} t}-\frac{b^{2}}{2 \sigma^{2}(T-t)}\right) \mathrm{d} t .
\end{aligned}
$$

The probability that a Wiener process starting at $a$ at time zero and ending up at $b$ at time $T$ hits zero during the time interval $[0, T]$ is obtained by integrating the conditional density $P\left(T^{a} \in \mathrm{d} t \mid W_{T}^{a}=b\right)$ over $[0, T]$, that is

$$
P\left(T^{a} \leq T \mid W_{T}^{a}=b\right)=\frac{a}{\sigma} \sqrt{\frac{T}{2 \pi}} \exp \left(\frac{(a-b)^{2}}{2 \sigma^{2} T}\right) \int_{0}^{T} \frac{\exp \left(-\frac{a^{2}}{2 \sigma^{2} t}-\frac{b^{2}}{2 \sigma^{2}(T-t)}\right)}{\sqrt{(T-t) t^{3}}} \mathrm{~d} t .
$$


Using Laplace transforms it is straightforward to show that, for positive $a$ and $b$,

$$
\int_{0}^{T} \frac{\exp \left(-\frac{a^{2}}{2 \sigma^{2} t}-\frac{b^{2}}{2 \sigma^{2}(T-t)}\right)}{\sqrt{(T-t) t^{3}}} \mathrm{~d} t=\frac{\sigma}{a} \sqrt{\frac{2 \pi}{T}} \exp \left(-\frac{(a+b)^{2}}{2 \sigma^{2} T}\right),
$$

and hence, since the sign of $b$ might be either positive or negative,

$$
P\left(T^{a} \leq T \mid W_{T}^{a}=b\right)= \begin{cases}\exp \left(-\frac{2 a b}{\sigma^{2} T}\right), & \text { if } b \geq 0 \\ 1, & \text { if } b<0\end{cases}
$$

where the statement for $b<0$ is obvious. This theoretical result is an important ingredient in the algorithm below and we note that it has been used before in numerical algorithms for reflected and stopped stochastic differential equations, see for example Ref. [12].

To obtain an efficient algorithm for the boundary behaviour of the stochastic model, we need a good estimate of the hitting times at the boundary. It is hard to sample hitting times directly from the density (2.11), but, fortunately, we can obtain an analytical expression for the expected first hitting time conditioned on the fact that the boundary is hit during $[0, T]$. From (2.11) and (2.14), we obtain

$$
\begin{aligned}
E\left[T^{a} \mid W_{T}^{a}=b, T^{a} \leq T\right] & =\int_{0}^{T} t P\left[T^{a} \in \mathrm{d} t \mid W_{T}^{a}=b, T^{a} \leq T\right]=\int_{0}^{T} t \frac{P\left(T^{a} \in \mathrm{d} t \mid W_{T}^{a}=b\right)}{P\left(T^{a} \leq T \mid W_{T}^{a}=b\right)} \\
& =\frac{a}{\sigma} \sqrt{\frac{T}{2 \pi}} \exp \left(\frac{(a+|b|)^{2}}{2 \sigma^{2} T}\right) \int_{0}^{T} \frac{\exp \left(-\frac{a^{2}}{2 \sigma^{2} t}-\frac{b^{2}}{2 \sigma^{2}(T-t)}\right)}{\sqrt{(T-t) t}} \mathrm{~d} t .
\end{aligned}
$$

Using Laplace transforms it is straightforward to show that, for positive $a$ and $b$,

$$
\int_{0}^{T} \frac{\exp \left(-\frac{a^{2}}{2 \sigma^{2} t}-\frac{b^{2}}{2 \sigma^{2}(T-t)}\right)}{\sqrt{(T-t) t}} \mathrm{~d} t=\pi \operatorname{erfc}\left(\frac{a+b}{\sqrt{2 \sigma^{2} T}}\right),
$$

where erfc is the complementary error function. Hence

$$
E\left[T^{a} \mid W_{T}^{a}=b, T^{a} \leq T\right]=\frac{a}{\sigma} \sqrt{\frac{\pi T}{2}} \exp \left(\frac{(a+|b|)^{2}}{2 \sigma^{2} T}\right) \operatorname{erfc}\left(\frac{a+|b|}{\sqrt{2 \sigma^{2} T}}\right) .
$$

Note here that with the variables $u=a / \sqrt{2 \sigma^{2} T}$ and $v=|b| / \sqrt{2 \sigma^{2} T}$, the expected first hitting time can be compactly expressed as

$$
E\left[T^{a} \mid W_{T}^{a}=b, T^{a} \leq T\right]=T u \sqrt{\pi} \operatorname{erfcx}(u+v),
$$

where erfcx is the scaled complementary error function. Since $\sqrt{\pi} \operatorname{erfcx}(x)<1 / x$ for all $x>0$, the expected first hitting time is always smaller than the hitting time obtained by using linear interpolation based on $a$ and $b$. Moreover, $\sqrt{\pi} \operatorname{erfcx}(x)$ $\rightarrow 1 / x$ as $x \rightarrow \infty$, so asymptotically the expected first hitting time coincides with the hitting time obtained by linear interpolation.

\subsection{First hitting times for the stochastic model}

Equipped with the results in Section 2.1, we are now ready to analyse the process $X_{t_{n}+t}$ confined to an interval $\left[L_{1}, L_{2}\right]$. The following arguments correspond to the case $L_{1}=0$ and $L_{2}=\infty$, but can easily be generalized to any $L_{1}$ and $L_{2}$ satisfying $L_{1}<L_{2}$. Consequently, the algorithm in Section 3 is stated in the setting of general $L_{1}$ and $L_{2}$. In the following we let $\left[t_{n}, t_{n+1}\right]$ denote a time step whose length $\Delta t_{n}:=t_{n+1}-t_{n}$ is of the order $10 \tau$.

We first investigate if $X_{t_{n}+t}<0$ for some $t \in[0,4 \tau]$, that is for values of $t$ for which the large $t$ approximation does not apply. Let $\varepsilon:=10 \tau \sqrt{e_{s}}$ so that $\varepsilon$, according to (2.5), exceeds two and a half standard deviations of $X_{t_{n}+4 \tau}$. If $X_{t_{n}}+M_{t_{n}} \tau<\varepsilon$, there is a non-negligible probability that $X_{t_{n}+t}$ has left the domain during the interval $[0,4 \tau]$. In that case, we will not be able to resolve the boundary behaviour with a single time step whose length is significantly larger than $\tau$. Instead, we can run a simulation with the scheme of Ref. [6] with time steps significantly smaller than $\tau$ and investigate if $X_{t_{n}+t}<0$ at the end of any of these smaller time steps. If so, we can use linear interpolation to estimate the exit time $T_{e}$ from the domain. The simulation for $t>T_{e}$ is described at the end of this section.

If $X_{t_{n}}+M_{t_{n}} \tau>\varepsilon$, then we simulate a value of $\left(X_{t_{n+1}}, M_{t_{n+1}}\right)$ based on the scheme of Ref. [4]. If $X_{t_{n+1}}<0$, then we know with certainty that the process has crossed the boundary. But also for $X_{t_{n+1}}>0$, there is a non-zero probability that the process has left the domain. Based on (2.8) and (2.14), the probability that $X_{t_{n}+t}<0$ for some $t \in\left(0, \Delta t_{n}\right)$ is

$$
\exp \left(-\frac{3 X_{t_{n+1}}\left(X_{t_{n}}+M_{t_{n}} \tau\right)}{\left(2 \Delta t_{n}-3 \tau\right) e_{s} \tau}\right)
$$


Hence, using this probability, we can determine whether $X_{t_{n}+t}$ has hit the boundary during the time step. If this is the case, we may use (2.18) to calculate the following estimate of the hitting time

$$
T_{e}=t_{n}+\frac{3 \tau}{2}+T \frac{x}{\sqrt{2 \sigma^{2} T}} \sqrt{\pi} \operatorname{erfcx}\left(\frac{x+|y|}{\sqrt{2 \sigma^{2} T}}\right),
$$

where $x=X_{t_{n}}+M_{t_{n}} \tau, y=X_{t_{n+1}}, T=\Delta t_{n}-3 \tau / 2$ and $\sigma=\sqrt{4 e_{s} \tau / 3}$. Note that the term $3 \tau / 2$ is added to the estimate of the first hitting time to compensate that the change of location of $X_{t}$ during a time interval of length $\Delta t_{n}$ is modelled by the change of location of a Wiener process during a time interval of length $\Delta t_{n}-3 \tau / 2$.

If the process has left the domain, either for small $t$ or for large $t$, we now have an estimate of the first exit time $T_{e}$. At the exit time, we sample a new velocity $M_{T_{e}}$ from a truncated Maxwellian distribution, see Ref. [4]. We use the scheme of Ref. [6] with small time steps to simulate the behaviour of the process during the remainder $\left[T_{e}, t_{n+1}\right]$ of the time step. If $X_{t}<0$ at the end of any of these smaller time steps, we use linear interpolation to determine the first exit time $T_{e}^{\prime}$, sample a new Maxwellian velocity and use the scheme of Ref. [6] with small time steps to simulate the behaviour of the process during the remainder $\left[T_{e}^{\prime}, t_{n+1}\right]$ of the time step. This procedure may have to be iterated a number of times.

To conclude this section, we discuss extensions to other types of reflecting boundary conditions. For specular reflection, one can simply use the algorithm of Ref. [4] with large time steps with the following correction. If $X_{t_{n+1}}$ is found outside the domain, it is orthogonally reflected into the domain and the sign of the velocity is altered. An extension to Maxwell boundary conditions is straightforward by combining specular and diffuse reflection with a proper accommodation coefficient.

\section{Algorithm}

In this section we describe the algorithm for generating the solution to (2.1)-(2.2) confined to an interval $\left[L_{1}, L_{2}\right]$ with diffuse reflection at the boundary. Let the length of the time step be $\Delta t_{n}=10 \tau$ and let $\tau, e_{s}, t_{n}, X_{t_{n}}$ and $M_{t_{n}}$ be given. Let $N$ be an integer with default value 200 . We assume that $L_{2}-L_{1} \gg 10 \tau \sqrt{4 e_{s} / 3}$, so that the probability that a particle travels from one boundary to the other in only one time step is insignificant (see step 5 below). Note that we have used $X_{t_{n}}, M_{t_{n}}$ and $\Delta t_{n}$ as variables in the pseudocode below and their values may change during the execution of the code.

(1) If $X_{t_{n}}+M_{t_{n}} \tau>L_{1}+10 \tau \sqrt{e_{s}}$ and $X_{t_{n}}+M_{t_{n}} \tau<L_{2}-10 \tau \sqrt{e_{s}}$, go to (3). Else let $t(k)=t_{n}+k \Delta t_{n} / N$ and go to (2).

(2) For $k=1: N$, generate $X_{t(k)}$ and $M_{t(k)}$ based on $X_{t(k-1)}$ and $M_{t(k-1)}$ using the scheme of Ref. [6] with time step $\Delta t_{n} / N$. If $X_{t(k)}<L_{1}$ for some $k \in\{1, \ldots, N\}$, let

$$
T_{e}=t_{n}+\frac{\Delta t_{n}}{N}\left(k+\frac{X_{t(k)}-L_{1}}{X_{t(k-1)}-X_{t(k)}}\right),
$$

and go to (8). If $X_{t(k)}>L_{2}$ for some $k \in\{1, \ldots, N\}$, let

$$
T_{e}=t_{n}+\frac{\Delta t_{n}}{N}\left(k+\frac{L_{2}-X_{t(k)}}{X_{t(k)}-X_{t(k-1)}}\right),
$$

and go to (9). Else go to (11)

(3) Generate $X_{t_{n+1}}$ and $M_{t_{n+1}}$ based on $X_{t_{n}}$ and $M_{t_{n}}$ using the scheme of Ref. [4] with time step $\Delta t_{n}$.

(4) If $X_{t_{n+1}}<L_{1}$, go to (6). If $X_{t_{n+1}}>L_{2}$, go to (7). Else go to (5).

(5) Generate a uniformly distributed random variable $V$ on $[0,1]$. If

$$
V<\exp \left(-\frac{3\left(X_{t_{n+1}}-L_{1}\right)\left(X_{t_{n}}+M_{t_{n}} \tau-L_{1}\right)}{\left(2 \Delta t_{n}-3 \tau\right) e_{s} \tau}\right),
$$

go to (6). Else if

$$
V<\exp \left(-\frac{3\left(L_{2}-X_{t_{n+1}}\right)\left(L_{2}-X_{t_{n}}-M_{t_{n}} \tau\right)}{\left(2 \Delta t_{n}-3 \tau\right) e_{s} \tau}\right)
$$

(6) Let

go to (7). Else go to (11).

$$
T_{e}=t_{n}+\frac{3 \tau}{2}+T \frac{x}{\sqrt{2 \sigma^{2} T}} \sqrt{\pi} \operatorname{erfcx}\left(\frac{x+|y|}{\sqrt{2 \sigma^{2} T}}\right),
$$

(7) Let

with $x=X_{t_{n}}+M_{t_{n}} \tau-L_{1}, y=X_{t_{n+1}}-L_{1}, T=\Delta t_{n}-3 \tau / 2$ and $\sigma=\sqrt{4 e_{s} \tau / 3}$. Go to (8).

$$
T_{e}=t_{n}+\frac{3 \tau}{2}+T \frac{x}{\sqrt{2 \sigma^{2} T}} \sqrt{\pi} \operatorname{erfcx}\left(\frac{x+|y|}{\sqrt{2 \sigma^{2} T}}\right),
$$

with $x=L_{2}-X_{t_{n}}-M_{t_{n}} \tau, y=L_{2}-X_{t_{n+1}}, T=\Delta t_{n}-3 \tau / 2$ and $\sigma=\sqrt{4 e_{s} \tau / 3}$. Go to (9). 


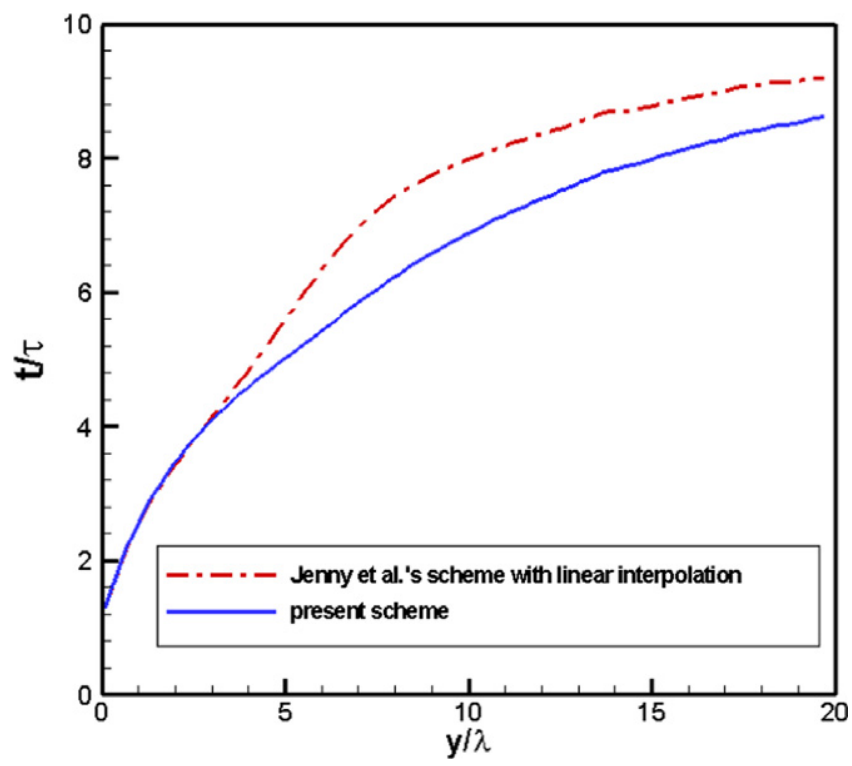

Fig. 1. Average first hitting time $t$ for particles initially found at a distance $y$ from the boundary as predicted by the scheme in Ref. [4] with linear interpolation and by the scheme presented in Section 3, respectively.

(8) Generate $M_{T_{e}}>0$ from a Maxwellian distribution and go to (10).

(9) Generate $M_{T_{e}}<0$ from a Maxwellian distribution and go to (10).

(10) Let $l$ be the smallest integer being greater than or equal to the quotient $N\left(t_{n+1}-T_{e}\right) / \Delta t_{n}$, let $t(k)=T_{e}+k\left(t_{n+1}-T_{e}\right) / l$, define $X_{t(0)}=L_{1}$ and $M_{t(0)}=M_{T_{e}}$. For $k=1: l$, generate $X_{t(k)}$ and $M_{t(k)}$ based on $X_{t(k-1)}$ and $M_{t(k-1)}$ using the scheme of Ref. [6] with time step $\left(t_{n+1}-T_{e}\right) / l$. If $X_{t(k)}<L_{1}$ for some $k \in\{1, \ldots, l\}$, let

$$
T_{e}^{\prime}=T_{e}+\frac{t_{n+1}-T_{e}}{l}\left(k+\frac{X_{t(k)}-L_{1}}{X_{t(k-1)}-X_{t(k)}}\right),
$$

let $T_{e}=T_{e}^{\prime}$ and go to (8). If $X_{t(k)}>L_{2}$ for some $k \in\{1, \ldots, l\}$, let

$$
T_{e}^{\prime}=T_{e}+\frac{t_{n+1}-T_{e}}{l}\left(k+\frac{L_{2}-X_{t(k)}}{X_{t(k)}-X_{t(k-1)}}\right),
$$

let $T_{e}=T_{e}^{\prime}$ and go to (9). Else go to (11).

(11) Save the values of $X_{t_{n+1}}$ and $M_{t_{n+1}}$ and use them as input during the next time step of length $10 \tau$ (that is go to (1)).

\section{Simulations}

In this section, we simulate a gas of Argon molecules confined to a one-dimensional box using the algorithm presented in Section 3. The initial state of the gas is given by standard conditions, that is, the temperature is $273 \mathrm{~K}$ and the pressure is $1 \mathrm{~atm}$. The length of the box is $1000 \lambda$, where $\lambda$ is the mean free path of gas molecules, and this corresponds to Knudsen number 0.001 . The wall temperature is fixed at $273 \mathrm{~K}$. Diffusive reflections are assumed at the boundary wall, meaning that molecules colliding with the wall rebound with a half-range Maxwellian distribution at the temperature of the corresponding wall. In order to obtain the distribution of macroscopic quantities, the simulation domain is divided into 300 cells, and each cell is assigned 500 molecules at the initial state. The calculating time step is $10 \tau$, where $\tau$ is the relaxation time.

Firstly, we compare the average first hitting time predicted by the scheme presented in Section 3 to the average first hitting time predicted by the scheme in Ref. [4] with linear interpolation. To obtain this comparison, we proceed as follows. In each calculating time step, if a molecule collides with one of the walls we record the hitting times predicted by our scheme and the distance $y$ between the previous location of the molecule and the hitting wall. In addition, we record the virtual hitting time obtained by linear interpolation method according to the previous location and the virtual new location. After $10^{3}$ simulation steps, the hitting times corresponding to a specific distance $y$ are averaged. Fig. 1 demonstrates how the expected first hitting time during time steps of length $10 \tau$ depends on the initial distance between the particle and the boundary and Fig. 2 shows the number of samples used for determining the first hitting times in Fig. 1. Note that the number of molecules colliding with the wall decreases as the distance $y$ to the wall increases.

For small $y$, the particles start close to the boundary and the scheme in Section 3 then uses small time steps with linear interpolation to determine the first hitting time. Hence the two curves in Fig. 1 coincide in the limit of small $y$. Note that the expected first hitting time does not converge to zero as $y$ tends to zero. This, perhaps surprising, result is due to half of the 


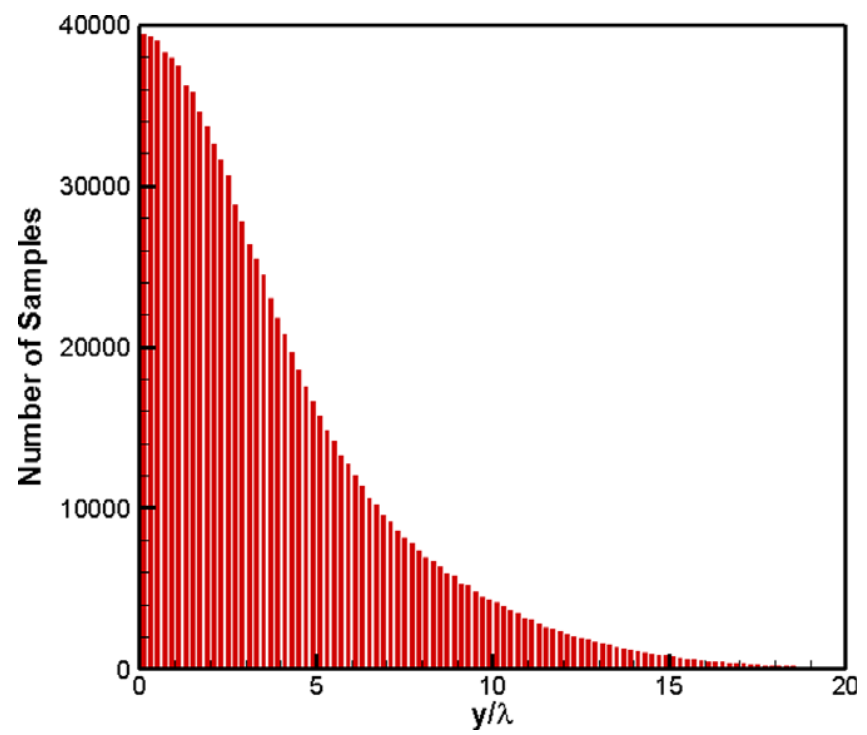

Fig. 2. Histogram of the number of samples used for determining the first hitting time for different values of the distance $y$ to the boundary.

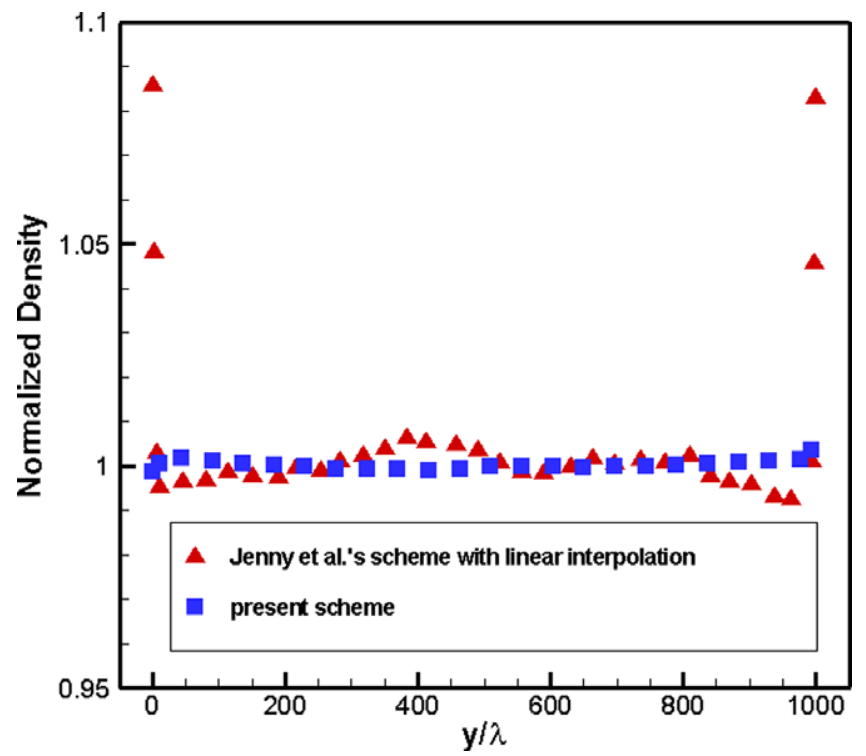

Fig. 3. Normalized density of molecules as predicted by the scheme in Ref. [4] with linear interpolation and by the scheme presented in Section 3 , respectively.

particles starting with a velocity directed away from the boundary. The motion of the particles for very small time steps is almost deterministic along the initial velocity and a strictly positive period of time will therefore elapse before the particles with initial velocity pointing away from the boundary will turn towards the boundary and hit it.

For larger $y$, Fig. 1 shows that the hitting time predicted by the scheme in Ref. [4] with linear interpolation exceeds the hitting time predicted by the scheme by the linear interpolation method is larger than that predicted by the scheme in Section 3. This is due to the molecular movement being a Wiener process rather than a linear process in the limit of large time steps. Note that the difference between the two estimates of the first hitting time has a maximum at approximately $8 \lambda$ and then decreases as $y$ is increased. The decreasing difference must occur since in the limit of infinite $y$ both estimates should be $10 \tau$. Note also that, as seen in Fig. 2, molecules which are initially found further than $20 \lambda$ away from a wall are very unlikely to collide with the wall in a single time step. Therefore, these particles are excluded from the plot in Fig. 1.

Fig. 3 shows the distribution of molecules along the one-dimensional box. The results are obtained by first simulating $10^{3}$ time steps and then averaging over the next $10^{3}$ time steps. Consequently, the number of samples for each cell is about $5 \cdot 10^{5}$ and the corresponding fractional error is about $1.4 \cdot 10^{-3}$ according to standard statistical mechanics [13]. Since the wall temperature and the initial gas temperature coincide, we expect the molecules to be uniformly distributed in this case. 
Indeed, the algorithm presented in Section 3 predicts a uniform distribution, with the variation between different locations in the box being less than $0.5 \%$. On the other hand, the scheme in Ref. [4], which uses linear interpolation to estimate the hitting time, clearly overestimates the density near the boundary walls, as shown in Fig. 1. This effect is due to linear interpolation overestimating the hitting time, as was described in the introduction.

\section{Conclusions}

The numerical scheme presented in Section 3 is shown to be an efficient stochastic particle Fokker-Planck algorithm with large time steps for wall-confined flows. The scheme at hand proves to be much more accurate close to the wall compared to the scheme of Jenny et al. [4] with only a slight increase in computational cost. There are schemes with small time steps, such as that of Gorji and Jenny [6], which obtain similar results close to the wall as the scheme presented in this article, but these schemes are much less computational efficient. To conclude, the scheme at hand predicts the correct near wall behaviour with a minimum of computational cost.

The authors are currently working on applying the algorithm presented in Section 3 to a variety of gas flows. Note that we present here only a one-dimensional algorithm, but the scheme could in the future be extended to two-dimensional and three-dimensional gas flows as well. Since our scheme is applicable for large time steps, it makes the simulation of large-scale gas flows using particle methods possible.

\section{Acknowledgements}

Jun Zhang was funded partly by the Engineering and Physical Sciences Council (EPSRC) of the UK under grant EP/I011927/1 and partly by the National Natural Science Foundation of China (Grant Nos. 11002147 and 11372325 ). Simulation results were obtained using the ARCHIE-WeSt High Performance Computer (www.archie-west.ac.uk), under EPSRC grant EP/K000586/1.

\section{References}

[1] Graeme A. Bird, Molecular Gas Dynamics and Direct Simulation of Gas Flows, Clarendon, Oxford, 1994.

[2] Francis J. Alexander, Alejandro L. Garcia, Berni J. Alder, Cell size dependence of transport coefficients in stochastic particle processes, Phys. Fluids 10 (1998) 1540-1542.

[3] Alejandro L. Garcia, Wolfgang Wagner, Time step truncation error in direct simulation Monte Carlo, Phys. Fluids 12 (2000) $2621-2633$.

[4] Patrick Jenny, Manuel Torrilhon, Stefan Heinz, A solution algorithm for the fluid dynamics based on a stochastic model for molecular motion, J. Comput. Phys. 229 (2010) 1077-1098.

[5] Fei Fei, Jing Fan, A diffusion information preservation method for small Knudsen number flows, J. Comput. Phys. 243 (2013) $179-193$.

[6] Mohammad H. Gorji, Patrick Jenny, An efficient Fokker-Planck algorithm for rarified gas flows, J. Comput. Phys. 262 (2014) 325-343.

[7] Mohammad H. Gorji, Maniel Torrilhon, Patrick Jenny, Fokker-Planck model for computational studies of monatomic rarified gas flows, J. Fluid Mech. 680 (2011) 574-601.

[8] Jun Zhang, Jing Fan, Langevin simulation of gas flows, in: Jing Fan (Ed.), Proceedings of the 28th International Symposium on Rarified Gas Dynamics, AIP Conference Proceedings, Xi'an, China, 2014.

[9] Jun Zhang, Dandan Zeng, Jing Fan, Analysis of transport properties determined by Langevin dynamics using Green-Kubo formulae, Physica A 411 (2014) 104-112.

[10] Stefan Heinz, Molecular to fliud dynamics: the consequences of stochastic molecular motion, Phys. Rev. E 70 (2004) 036308.

[11] Stefan Heinz, Comment on analysis of transport properties determined by Langevin dynamics using Green-Kubo formulae, Physica A 423 (2015) $27-32$.

[12] Emmanuel Gobet, Euler schemes and half-space approximation for the simulation of diffusion in a domain, ESAIM Probab. Stat. 5 (2001) $261-297$.

[13] Nicolas G. Hadjiconstantinou, Alejandro L. Garcia, Martin Z. Bazant, Gang He, Statistical error in particle simulations of hydrodynamic phenomena, J. Comput. Phys. 187 (2003) 274-297. 